\title{
PCR amplification of the erm(41) gene can be used to predict the sensitivity of Mycobacterium abscessus complex strains to clarithromycin
}

\author{
AYAKA MASE $^{1,2}$, FUMIHIRO YAMAGUCHI ${ }^{1,2}$, TOSHITAKA FUNAKI $^{2}$, YOHEI YAMAZAKI $^{2}$, \\ YUSUKE SHIKAMA $^{2}$ and KUNIHIKO FUKUCHI ${ }^{1}$ \\ ${ }^{1}$ Department of Clinical Pathology, Showa University School of Medicine, Tokyo 142-8666; \\ ${ }^{2}$ Department of Respiratory Medicine, Showa University Fujigaoka Hospital, Yokohama, Kanagawa 227-8501, Japan
}

Received November 23, 2018; Accepted May 8, 2019

DOI: $10.3892 /$ etm.2019.8289

\begin{abstract}
A worldwide increase in the Mycobacterium abscessus (M. abscessus) complex has been observed. Therefore, the aim of the present study was to investigate the diversity of the $r r l$ and $\operatorname{erm}(41)$ genes, both of which are associated with macrolide sensitivity in the M. abscessus complex. The current study also examined the efficacy of mass spectrometry as an alternative to molecular testing to classify subspecies of the M. abscessus complex. A total of 14 strains of the M. abscessus complex were obtained, and based on conventional analyses using housekeeping genes, $57 \%$ were determined to be M. abscessus subsp. abscessus, $43 \%$ were M. abscessus subsp. massiliense, and none were identified as M. abscessus subsp. bolletii. However, depending on the strain, it was not always possible to distinguish between the subspecies by mass spectrometry. Consequently, PCR products for the $r r l$ and $\operatorname{erm}$ (41) genes were directly sequenced. Overall, $7.1 \%$ of the strains were identified to have a $r r l$ mutation, and $92.9 \%$ carried a $\mathrm{T}$ at position 28 of erm(41). Results presented here suggest that the principal cause of treatment failure for M. abscessus complex infections is inducible macrolide resistance encoded by the erm(41) gene. From a strictly pragmatic standpoint, the phenotypic function of a putative erm(41) gene is the most important piece of information required by clinicians in order to prescribe an effective treatment. Although PCR amplification of erm(41) is not sufficient to differentiate between the M. abscessus complex subspecies, PCR can be easily and efficiently used to predict the sensitivity of members of the M. abscessus complex to clarithromycin.
\end{abstract}

Correspondence to: Dr Ayaka Mase, Department of Clinical Pathology, Showa University School of Medicine, 1-5-8 Hatanodai, Tokyo 142-8666, Japan

E-mail: ayaka0507@med.showa-u.ac.jp

Key words: Mycobacterium abscessus complex, rrl, erm(41), matrix-assisted laser desorption ionization-time of flight mass spectrometry, macrolide resistance

\section{Introduction}

Recent epidemiology research has revealed a worldwide increase in non-tuberculous mycobacteria (NTM) infections. In Japan in particular, the Mycobacterium abscessus (M. abscessus) complex is the third most common pathogen in pulmonary diseases caused by NTM, after the Mycobacterium avium complex and Mycobacterium kansasii (1). The M. abscessus complex is categorized as rapidly growing mycobacteria, defined by visible growth within seven days, and is one of the most difficult pathogens to treat. Over the past decade, the M. abscessus complex has been subclassified into three new subspecies: M. abscessus subsp. abscessus, M. abscessus subsp. massiliense and M. abscessus subsp. bolletii (2). Macrolides are the key drugs used for the treatment of M. abscessus complex infection; however, macrolides are not always effective or in some cases they lose effectiveness during the course of treatment. Acquired macrolide resistance is associated with point mutations in the $r r$ gene, which encodes $23 \mathrm{~S}$ rRNA (3). An erythromycin ribosomal methylase, encoded by erm(41) in the M. abscessus complex, confers inducible resistance to macrolides (4). The functionality of the erm(41) gene differs depending on the subspecies. Most notably, M. abscessus subsp. massiliense has been proposed to have an incomplete erm(41) gene, which is associated with macrolide sensitivity. In addition, some M. abscessus subsp. abscessus strains have substitutions in the erm(41) gene that also lead to macrolide susceptibility. Thus, it is important to distinguish the three kinds of subspecies and to analyze the sequences of the $r r l$ and erm(41) genes. Matrix-assisted laser desorption ionization-time of flight mass spectrometry (MALDI-TOF MS) has been used for microbial identification in recent years, and several researchers have attempted to apply this tool to differentiate the subspecies of the M. abscessus complex. However, different diagnostic criteria have been used at different institutions and the results of the method are inconsistent (5-10).

Few studies have investigated the ratio of subspecies of the M. abscessus complex in Japan, or examined their macrolide resistance genes (11). It is likely that regional differences in the ratios of the subspecies and the clinical features of such isolates may exist. In the present study, we aimed to examine the sequence of the erm(41) gene in M. abscessus complex 
subspecies. We also compared the efficacy of using molecular testing and mass spectrometry to classify subspecies of the M. abscessus complex.

\section{Materials and methods}

Samples and data collection. Fourteen strains of the M.abscessus complex were obtained from each patient between July 2016 and April 2018 at Showa University Hospital (Tokyo) or at Showa University Fujigaoka Hospital (Yokohama). For reference, one strain of Mycobacterium fortuitum (M. fortuitum) was collected during the period. All strains were of sputum origin except for one M. abscessus complex isolate from a bronchoscopy. Clinical isolates were cultured in mycobacteria growth indicator tubes (MGIT) and in 2\% Ogawa solid medium. M.abscessus complex and M.fortuitum were distinguished by DNA-DNA hybridization. All clinical data were collected from medical records. Official approval for the study was obtained in advance from the Ethics Committee for Research at Showa University (approved numbers 371 and 2016127). Informed consent was waived because of the retrospective nature of the study.

Molecular testing. DNA was extracted from mycobacterial clinical isolates using InstaGene matrix (Bio-Rad Laboratories) and stored at $-20^{\circ} \mathrm{C}$. The amount of DNA extracted ranged from 104 to $452 \mathrm{ng} / \mu \mathrm{l}$. Primers for nucleic acid amplification were designed as indicated in Table I. PCRs were performed to amplify mutation hot spot regions in the housekeeping genes $h s p 65$, rpoB and ITS to classify the strains into the three subspecies using a Mycycler ver.10.65 thermal cycler (Bio-Rad Laboratories). The $r r l$ and $\operatorname{erm}(41)$ genes were also amplified in a similar manner. All PCR assays were carried out in $25-\mu 1$ volumes containing $200 \mathrm{ng}$ of template DNA, 0.1 units of Taq DNA polymerase (Roche Diagnostics), 25 pmol of each primer, and $10 \mathrm{nmol}$ of dNTPs. Cycling parameters were $30 \mathrm{sec}$ at $95^{\circ} \mathrm{C}, 30 \mathrm{sec}$ at $60^{\circ} \mathrm{C}$, and $60 \mathrm{sec}$ at $72^{\circ} \mathrm{C}$ for 30 cycles. PCR products were separated on a $5 \%$ polyacrylamide gel or $1 \%$ agarose gel. The gels were stained with ethidium bromide and photographed under UV illumination. The PCR products were purified and were directly sequenced using a BigDye terminator kit and ABI Prism $3130 x l$ (Applied Biosystems). When sequences could not be obtained by direct sequencing, the PCR products were ligated into a pGEM T easy vector (Promega), which was then used to transform JM109 cells, as reported previously (12). Multiple clones were selected and plasmid DNA was purified from each and sequenced. The reference sequences for each gene were obtained from GenBank (accession numbers CU458896.1: M. abscessus subsp. abscessus, AP_014547.1: M. abscessus subsp. massiliense.).

Antibiotic susceptibility test. Minimum inhibitory concentrations (MICs) of amikacin and clarithromycin were determined by the broth microdilution method and were interpreted according to the Clinical and Laboratory Standards Institute document M24-A2 (13). Briefly, an appropriate volume of the culture was transferred into $3 \mathrm{ml}$ of sterilized saline until the turbidity matched that of a $0.5 \mathrm{McF}$ arland standard. A $10 \mu 1$ aliquot of the suspension was used to inoculate $11 \mathrm{ml}$ of cation-adjusted Mueller-Hinton medium and $100 \mu 1$ was distributed into each of the 96 well panels. The panels were incubated for $72 \mathrm{~h}$ at $30^{\circ} \mathrm{C}$, and growth was determined. To test for inducible resistance to clarithromycin, the MICs for clarithromycin were also determined after 7 and 14 days of incubation.

Mass spectrometry. Colonies were transferred into microcentrifuge tubes containing $300 \mu \mathrm{l}$ of sterile deionized water, and the tubes were incubated for $30 \mathrm{~min}$ at $95^{\circ} \mathrm{C}$. Then samples were mixed with $900 \mu 1$ of $70 \%$ ethanol by vortexing for $1 \mathrm{~min}$. The suspensions were centrifuged at 13,000 rpm for $2 \mathrm{~min}$, and the pellets were dried for $5 \mathrm{~min}$ at room temperature and resuspended in $20 \mu \mathrm{l}$ of $100 \%$ acetonitrile with zirconia beads. The mixtures were vortexed for $1 \mathrm{~min}$. The samples were then suspended with $20 \mu 1$ of $70 \%$ formic acid and centrifuged at $13,000 \mathrm{rpm}$ for $2 \mathrm{~min}$. Subsequently, $1 \mu \mathrm{l}$ of the supernatant from each extract was spotted on a target plate. After drying, $1 \mu \mathrm{l}$ of matrix solution (saturated $\alpha$-cyano-4-hydroxycinnamic acid in $47.5 \%$ acetonitrile and $2.5 \%$ trifluoroacetic acid) was added onto each spot. Mass spectra were obtained on a MALDI Biotyper ver 4.0 configured with Micro flex LT/SH with Mycobacteria Library ver.5.0 (Bruker Daltonik). Spectra were analyzed by Flex Analysis software 3.4 and MBT compass explore ver 4.1 (Bruker Daltonik).

Statistical analysis. The significance in each group was evaluated with Fisher's exact test or Pearson's Chi-square test, unpaired student's t-test, and the nonparametric Mann-Whitney test on ranks. $\mathrm{P}<.05$ was considered significant. All analyses were performed using JMP 13.0 software (SAS Institute).

\section{Results}

Determination of M. abscessus complex subspecies by sequencing housekeeping genes. The results of sequence analyses of housekeeping genes are shown in Table II. To distinguish the three subspecies, $h s p 65, r p o B$ and ITS sequences were determined by direct sequencing and compared to reference sequences. The sequences of the $h s p 65$ genes from eight strains were consistent with the reference sequence from M. abscessus subsp. abscessus, while those from six strains were consistent with the $h s p 65$ reference sequence from M. abscessus subsp. massiliense, with the exception of one strain (no. 9626), which had a change at position 280T $>$ A. High heterogeneity of $r p o B$ in the M. abscessus complex has been reported (14). The rpoB genes from eight strains were identical to the reference gene from M. abscessus subsp. abscessus, while a $37 \mathrm{C}>\mathrm{T}$ change was present in two strains (no. 71740 and no. 9614), and two changes $(52 \mathrm{C}>\mathrm{T}$ and $391 \mathrm{C}>\mathrm{T}$ ) were found in another strain (no. 8548). Six strains had $r p o B$ sequences identical to the reference sequence from M. abscessus subsp. massiliense, with the exception of one substitution, 316T $>C$ that was detected in four strains (nos. 74369, 77944, 9626, and 9388). No amino acid changes resulted from these nucleotide sequence differences. Together, eight strains were identified as M. abscessus subsp. abscessus, and six strains as M. abscessus subsp. massiliense. The results of sequence analyses of the ITS region were consistent with these findings. However, a novel insertion sequence (180_181GTTGT) was found in one strain of M. abscessus subsp. abscessus (no. 71740). 
Table I. Primer design.

\begin{tabular}{|c|c|c|c|}
\hline Target & Primers & Sequence & bp \\
\hline \multirow[t]{2}{*}{ hsp65 } & hsp65F & 5'-ACCAACGATGGTGTGTCCAT-3' & 441 \\
\hline & hsp65R & 5'-CTTGTCGAACCGCATACCCT-3' & \\
\hline \multirow[t]{2}{*}{ rров } & rpoBF & 5'-GAGGGTCAGACCACGATGAC-3' & 408 \\
\hline & rpoBR & 5'-AGCCGATCAGACCGATGTT-3' & \\
\hline \multirow[t]{2}{*}{ ITS } & ITSF & 5'-TTGTACACACCGCCCGTC-3' & 490 \\
\hline & ITS336R & 5'-СTTCTAGTGCCAAGGCATTCACC-3' & \\
\hline \multirow[t]{2}{*}{$r r l$} & $\operatorname{rrl} 2145 \mathrm{~F}$ & 5'-GCGAAATTCCTTGTCGGGTAAGT-3' & 283 \\
\hline & $\operatorname{rrl} 2427 \mathrm{R}$ & 5'-GGATATACGGTCCGAGGTTAG-3' & \\
\hline \multirow[t]{2}{*}{ erm(41) } & erm- $86 \mathrm{~F}$ & 5'-GACCGGGGCCTTCTTCGTGAT-3' & 673 \\
\hline & erm64R & 5'-GACTTCCCCGCACCGATTCC-3' & \\
\hline
\end{tabular}

bp, base pair.

Patients and characteristics. As mentioned above, results were obtained for all patients, $57 \%$ (8 of 14) of whom were infected with M. abscessus subsp. abscessus, and 43\% (6 of 14) of whom were infected with M. abscessus subsp. massiliense. None were infected with M. abscessus subsp. bolletii. Table III shows the patient characteristics. There were seven males and seven females whose ages at diagnosis ranged from 30 to 83 years: Thirteen were Japanese and one was Indian. According to the guidelines published by the American Thoracic Society/Infectious Diseases Society of America (15), all patients were newly diagnosed with $M$. abscessus complex pulmonary disease, based on at least two positive culture results derived from pulmonary samples. As shown in Table III, there was no significant association of the subspecies with age, body-mass index, sex, smoking history, radiological findings, hemoptysis, sputum smear, or C-reactive protein.

Gene status of $\mathrm{rrl}$ and erm(41). Sequence differences identified in the $r r l$ and erm(41) genes are summarized in Table IV. In the $r r l$ gene, a $A>G$ change was detected at position 2059 in one strain (no. 8006), but no other alterations were found. As for the erm(41) gene, nucleotides at positions 64_65 and 159_432 were deleted in strains of M. abscessus subsp. massiliense, compared to the M. abscessus subsp. abscessus strains. Eight substitutions were found in the M. abscessus subsp. abscessus isolates, whereas no substitutions were found in the strains of M. abscessus subsp. massiliense. In those isolates of M. abscessus subsp. abscessus, 28T $>C, 238 \mathrm{~A}>\mathrm{G}$ and $419 \mathrm{C}>\mathrm{T}$ substitutions were responsible for the amino-acid changes W10R, I80V and P140L, respectively. As shown in Fig. 1, the sizes of the PCR products amplified from the erm(41) genes were consistent with sequencing results ( 673 base pairs for M. abscessus subsp. abscessus, and 397 base pairs for M. abscessus subsp. massiliense), and identifications based on the size of the erm(41) gene were consistent with those based on $h s p 65, r p o B$ and ITS sequences.

Antimicrobial sensitivity. Table $\mathrm{V}$ shows the antibiotic susceptibility of the M. abscessus strains to amikacin and clarithromycin. The MICs of amikacin ranged from 2 to $16 \mu \mathrm{g} / \mathrm{ml}$, which indicated that all strains were sensitive.
There was no difference in the MICs between the two subspecies. M. abscessus subsp. abscessus isolates were sensitive to clarithromycin on day 3 , but the MICs were significantly higher on day 14 with one exception (no. 9944). In contrast, the strains of M. abscessus subsp. massiliense were susceptible to clarithromycin on days 3 through 14, except for one strain (no. 8006), which showed resistance from the start. Strain no. 9626 was sensitive early in the testing period, but the MIC was about 4-fold higher on day 14 .

MALDI-TOF MS analysis. The details of mass spectra are shown in Figs. 2 and 3. Out of the 14 isolates, 11 were analyzed using MALDI-TOF MS. For reference, one clinical isolate of $M$. fortuitum was simultaneously analyzed in a similar manner. The 11 strains were identified as M.abscessus complex (score range, 1.66 to 2.14) and were correctly differentiated from the strain of M. fortuitum (Fig. S1). As shown in Fig. 2, the representative spectra of M. abscessus complex subspecies were similar at a laser frequency of $50 \mathrm{~Hz}$ across 2,000 to $12,000 \mathrm{~m} / \mathrm{z}$. When magnifying the spectrum (Fig. 3), distinctive peaks reported previously $(8,10)$ were detected in some cases, but not in all samples. More specifically, peaks around 4390, 7639, 8781 and $9473 \mathrm{~m} / \mathrm{z}$ for M. abscessus subsp. abscessus and peaks around 4385, 7669, and $8767 \mathrm{~m} / \mathrm{z}$ for M. abscessus subsp. massiliense were found. However, each baseline was unstable and the peaks were wide and low for these strains. Moreover, discriminating peaks were mostly of low intensity or were overlapping. Thus, detection of clearly identifiable differences between strains of different subspecies was extremely difficult. Overall, it was possible to discriminate between M. abscessus subsp. abscessus and M. abscessus subsp. massiliense by using mass spectra only in some cases.

\section{Discussion}

In the current study, M. abscessus complex isolates obtained from patients treated at our institutes located in the Tokyo-Yokohama area were analyzed. Overall, 57\% (8 of 14) of the isolates were identified as M. abscessus subsp. abscessus, 43\% (6 of 14) as M. abscessus subsp. massiliense, and none as $M$. abscessus subsp. bolletii. It has been reported 


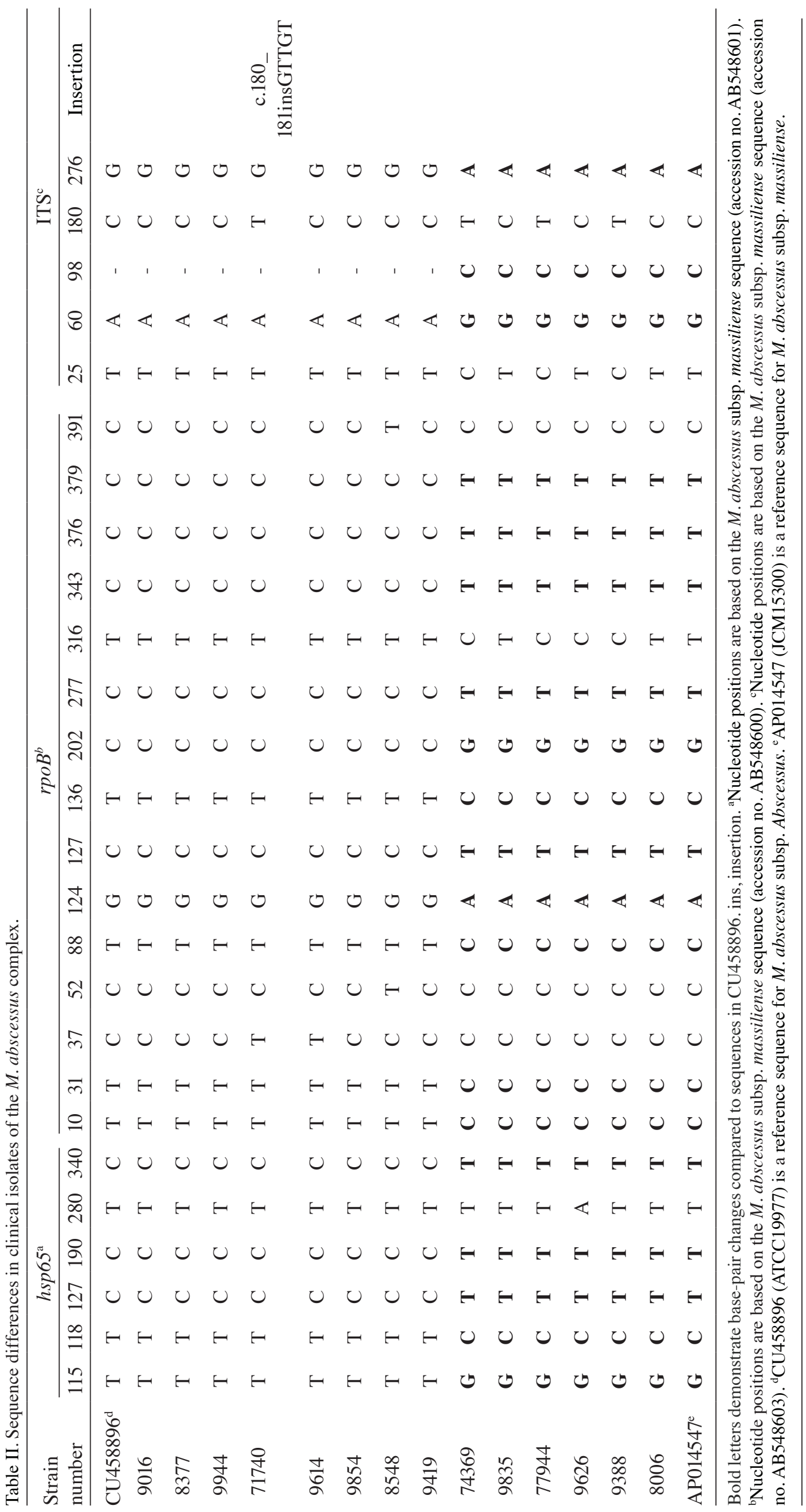


Table III. Characteristics of patients.

\begin{tabular}{|c|c|c|c|}
\hline Characteristics & $\begin{array}{l}\text { M. abscessus subsp. abscessus } \\
\qquad(\mathrm{n}=8)\end{array}$ & $\begin{array}{l}\text { M. abscessus subsp. massiliense } \\
\qquad(\mathrm{n}=6)\end{array}$ & $\mathrm{P}$-value \\
\hline Age (years) & $69.1 \pm 18.1$ & $62.5 \pm 9.7$ & 0.435 \\
\hline $\mathrm{BMI}, \mathrm{kg} / \mathrm{m}^{2}$ & $19.6 \pm 3.4$ & $18.6 \pm 1.8$ & 0.552 \\
\hline \multicolumn{4}{|l|}{ Sex } \\
\hline Male & $3(21.4)$ & $4(28.5)$ & \multirow[t]{2}{*}{0.592} \\
\hline Female & $5(35.7)$ & $2(14.2)$ & \\
\hline \multicolumn{4}{|l|}{ Smoking } \\
\hline Never & $6(42.8)$ & $2(14.2)$ & \multirow[t]{2}{*}{0.277} \\
\hline Ever & $2(14.2)$ & $4(28.5)$ & \\
\hline \multicolumn{4}{|c|}{ Radiological findings } \\
\hline Cavity & $1(7.1)$ & $2(14.2)$ & 0.538 \\
\hline \multicolumn{4}{|c|}{ Symptom of hemoptysis } \\
\hline Yes & $2(14.2)$ & $3(21.4)$ & \multirow[t]{2}{*}{0.58} \\
\hline No & $6(42.8)$ & $3(21.4)$ & \\
\hline \multicolumn{4}{|l|}{ Positive smear } \\
\hline Yes & $6(42.8)$ & $3(21.4)$ & \multirow[t]{2}{*}{0.58} \\
\hline No & $2(14.2)$ & $3(21.4)$ & \\
\hline \multicolumn{4}{|c|}{ Laboratory findings } \\
\hline $\mathrm{CRP}, \mathrm{mg} / \mathrm{dl}$ & $0.96 \pm 1.22$ & $1.68 \pm 2.17$ & 0.492 \\
\hline
\end{tabular}

Date are expressed as numbers $(\%)$, values are means \pm standard deviation. BMI, body mass index; CRP, C-reactive protein.

that M. abscessus subsp. abscessus is the most predominant subspecies of the complex followed by M. abscessus subsp. massiliense, and that M.abscessus subsp. bolletii is quite rare, ranging from 0-3\% in Japan (11,16-19). There were no significant differences in clinical features between $M$. abscessus subsp. abscessus and M. abscessus subsp. massiliense. Clinical characteristics of the M. abscessus complex did not help us to distinguish those subspecies, which is consistent with findings reported in the literature $(11,20)$.

The proportion of the M. abscessus complex subspecies varies depending on the region from which they are isolated. Compared to Western Europe, the prevalence of M. abscessus subsp. bolletii is lower in East Asian countries (21-24). Although, M. abscessus subsp. abscessus is the most predominant subspecies of the complex in most parts of the world, some studies have reported that M. abscessus subsp. massiliense is more abundant than M. abscessus subsp. abscessus in some parts of East Asia such as Taiwan and Korea $(5,21,22,25)$. Actually whole-genome sequencing analyses revealed genetic distinctions between M. abscessus subsp. abscessus isolates in Asia and Western Europe (26), suggesting that the phylogenetic diversity correlates with the regional ratio of the subspecies.

The current commercial system for NTM differentiation in Japan consists of the DNA-DNA hybridization method, which is unable to differentiate subspecies of the M. abscessus complex. The three subspecies also cannot be distinguished by 16S rRNA gene sequencing, which is commonly used for bacterial taxonomy in academic research, because the 16S rRNA genes in the three subspecies are $100 \%$ identical (27). The differentiation requires sequencing of several housekeeping genes, which is not easy to accomplish in most mycobacteriology laboratories. Hence, these three subspecies have not been distinguished in hospital laboratories. Sequencing of a single target gene may lead to inaccurate identification of closely related subspecies; however, multilocus sequence analyses of the $M$. abscessus complex have been described using hsp65, rpoB, ITS, gyrB, dnaA, recA and secA (28). Although some other methods based on technology developed by multilocus sequence analyses have been designed, such as variable-number tandem repeat analysis $(18,19,29,30)$ and multiplex PCR $(17,31)$, those methods are complicated. In the present study, subspecies of the M. abscessus complex were differentiated based on partial sequences of the $h s p 65$ and $r p o B$ genes, and results of ITS sequencing were also consistent in differentiating the two subspecies. In a subset of M. abscessus complex isolates, a hybrid genetic pattern for the hsp65 and rpoB genes has been reported $(32,33)$, presumably the result of horizontal gene transfer between the subspecies. In such cases ITS gene analysis was essential to identify the subspecies. Sequencing of at least three housekeeping genes should therefore be carried out for subspecies identification.

Numerous institutions are seeking an alternative way to distinguish the M. abscessus subspecies in clinical practice. MALDI-TOF MS has been evaluated for the identification of microorganisms including mycobacteria. However in this study, it was not possible to distinguish between the M. abscessus subspecies of all isolates by MALDI-TOF MS. Although several institutes have reported the efficacy of MALDI-TOF MS in differentiating the three subspecies of the M. abscessus complex (5-10), methods for sample 


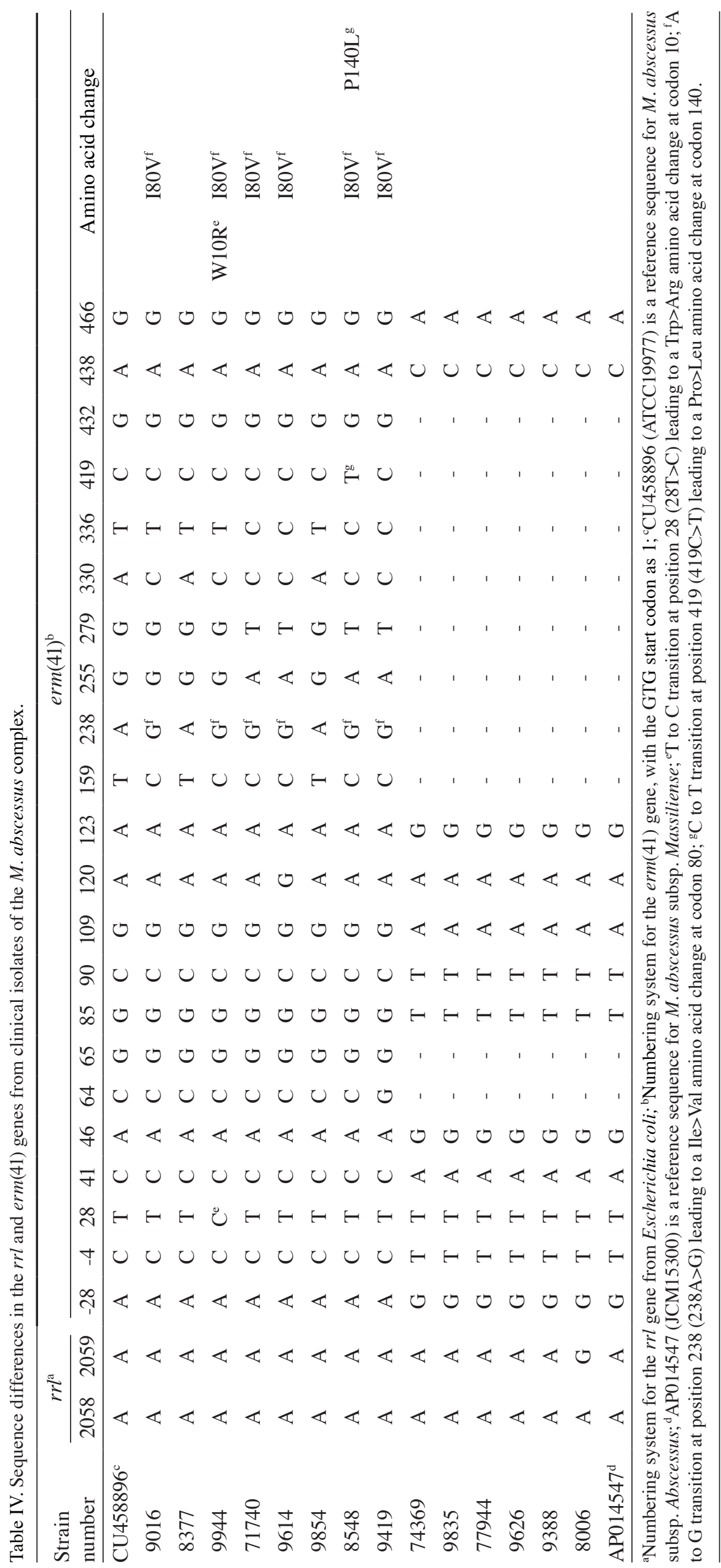




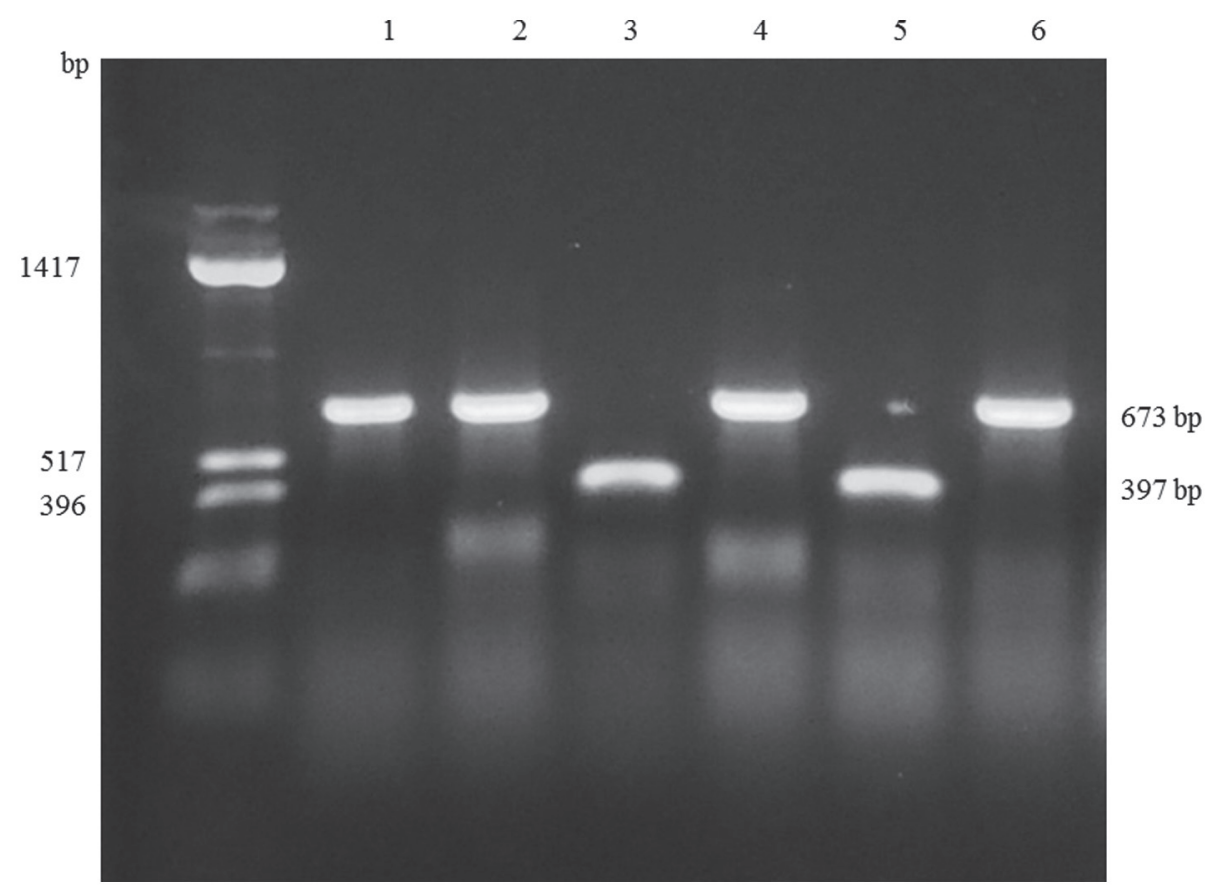

Figure 1. Representative PCR products for the erm(41) gene. The amplified products from M. abscessus subsp. abscessus strains (no. 9016, no. 8377, no. 9944 and no. 9854) were 673 bp in length, whereas those from M. abscessus subsp. massiliense strains (no. 9835 and no. 9626) were 397 bp in length. Far left lane, DNA size standard; Lane 1, no. 8377; Lane 2, 9016; Lane 3, 9626; Lane 4, 9944; Lane 5, 9835; Lane 6, 9854.

preparation and analysis, and diagnostic criteria have not been standardized. When defining the range from 2,000 to $20,000 \mathrm{~m} / \mathrm{z}$, ribosomal protein accounts for 50 to $70 \%$ of the peptide detected by MALDI-TOF MS. Thus, it is easy to distinguish between species that contain diverse ribosomal proteins. In fact, the current study revealed obvious differences between $M$. fortuitum and the M. abscessus complex by mass spectra (Fig. S1). However, the mass spectrometry peaks may be affected by variations in culture media formulations, duration of growth, and other conditions. The utility of MGIT liquid medium (10), 5\% sheep blood agar (6), Middlebrook $7 \mathrm{H} 11$ (7), and Lowenstein-Jensen agar (9) have been reported for MALDI-TOF MS. In the present study, samples were prepared from colonies incubated on $2 \%$ Ogawa solid medium. Thus, the diagnostic criteria for MALDI-TOF analysis differ depending on the laboratory carrying out the analyses. Since there are few differences in the ribosomal proteins between the M. abscessus complex subspecies, and the sample preparation for mass spectra is even affected by climate, the procedure is often poorly reproducible. Considerable effort would be necessary to optimize and standardize a protocol to obtain reproducible mass spectrometry results for differentiating the M. abscessus complex subspecies. A proteomics study revealed specific immunogenic proteins in the $M$. abscessus complex; therefore, the subspecies may be differentiated using immunoassays (34).

Mutations in $r r l$ gene confer acquired resistance and nucleotide T28 of erm(41) is associated with inducible resistance. Since most of the alterations identified in this study were in the $\operatorname{erm}(41)$ gene $(92.9 \% ; 13$ of 14 isolates) rather than the $r r l$ gene $(7.1 \% ; 1$ of 14$)$, we concluded that the main cause of treatment failure for M. abscessus complex infections was inducible resistance encoded by the erm(41) gene. One strain of
M. abscessus subsp. massiliense (no. 8006) harboring a point mutation $2059 \mathrm{~A}>\mathrm{G}$ in the $r r l$ gene showed resistance to clarithromycin from day 3 through day 14 (MIC $>128 \mu \mathrm{g} / \mathrm{ml}$ ). The presence of $r r l$ mutations was reported in up to $30 \%$ of newly isolated strains in East Asia $(16,35)$, whereas no $r r l$ mutations were identified at the time of diagnosis in Spain (36). It has been reported that mutations in the $\mathrm{rrl}$ gene rapidly accumulate following clarithromycin use in monotherapy (3). Conversely, several studies have reported on acquired resistance due to $\mathrm{rrl}$ mutations in the absence of any macrolide exposure $(16,37)$. However, an in vitro study reported that $r r l$ mutations at position 2058 or 2059 were observed during incubation with clarithromycin (38). In East Asia, low dose macrolides are often administered for prolonged periods to treat chronic respiratory disorders, such as diffuse panbronchiolitis, to stimulate an immunomodulatory effect (39). Hence, careful attention is needed when prescribing macrolides to patients initially diagnosed with pulmonary disease caused by M. abscessus complex. One strain of $M$. abscessus subsp. abscessus (no. 9944) carried a substitution at position $28 \mathrm{~T}>\mathrm{C}$ in the erm(41) gene, which resulted in an amino acid change (W10R), and showed a low MIC value for clarithromycin. Other strains that harbored amino acid substitutions at I80V $(238 \mathrm{~A}>\mathrm{G})$ or P140L (419C >T) showed inducible resistance. These data are in agreement with previous reports $(4,37)$ suggesting that the $5^{\prime}$ end of the erm(41) gene is a key region because this region is also predicted to carry a second open reading frame encoding a leader peptide that regulates expression of the erm(41) gene itself (4). In M. abscessus subsp. massiliense, two base deletions (61_62del) have been commonly detected, in addition to longer deletions (159_432del), which are consistent with the findings of the present study. Strains of M. abscessus subsp. abscessus harboring a $\mathrm{C}>\mathrm{T}$ substitution at position 19 , resulting in a stop 
Table V. Antibiotic susceptibilities of M.abscessus complex isolates based on MIC $(\mu \mathrm{g} / \mathrm{ml})$ values.

\begin{tabular}{|c|c|c|c|c|c|}
\hline \multirow[b]{2}{*}{ Isolate } & \multirow[b]{2}{*}{ Strains no. } & \multirow{2}{*}{$\frac{\text { Amikacin }}{\text { Day } 3}$} & \multicolumn{3}{|c|}{ Clarithromycin } \\
\hline & & & Day 3 & Day 7 & Day 14 \\
\hline \multirow[t]{8}{*}{ M. abscessus subsp. abscessus } & 9016 & 16 & 0.125 & 64 & 64 \\
\hline & 8377 & 16 & 0.5 & 64 & $>128$ \\
\hline & 9944 & 8 & $\leq 0.06$ & $\leq 0.06$ & $\leq 0.06$ \\
\hline & 71740 & 8 & 0.25 & 32 & 32 \\
\hline & 9614 & 8 & 0.25 & 64 & 64 \\
\hline & 9854 & 8 & 0.125 & $>128$ & $>128$ \\
\hline & 8548 & 8 & $\leq 0.06$ & 64 & $>128$ \\
\hline & 9419 & 8 & 0.5 & 32 & 32 \\
\hline \multirow[t]{6}{*}{ M. abscessus subsp. massiliense } & 74369 & 16 & 0.25 & 0.25 & 0.25 \\
\hline & 9835 & 16 & $\leq 0.06$ & $\leq 0.06$ & $\leq 0.06$ \\
\hline & 77944 & 4 & $\leq 0.06$ & $\leq 0.06$ & $\leq 0.06$ \\
\hline & 9626 & 16 & 0.125 & 0.25 & 0.5 \\
\hline & 9388 & 8 & $\leq 0.06$ & $\leq 0.06$ & $\leq 0.06$ \\
\hline & 8006 & 2 & $>128$ & $>128$ & $>128$ \\
\hline
\end{tabular}

MIC, minimum inhibitory concentration.

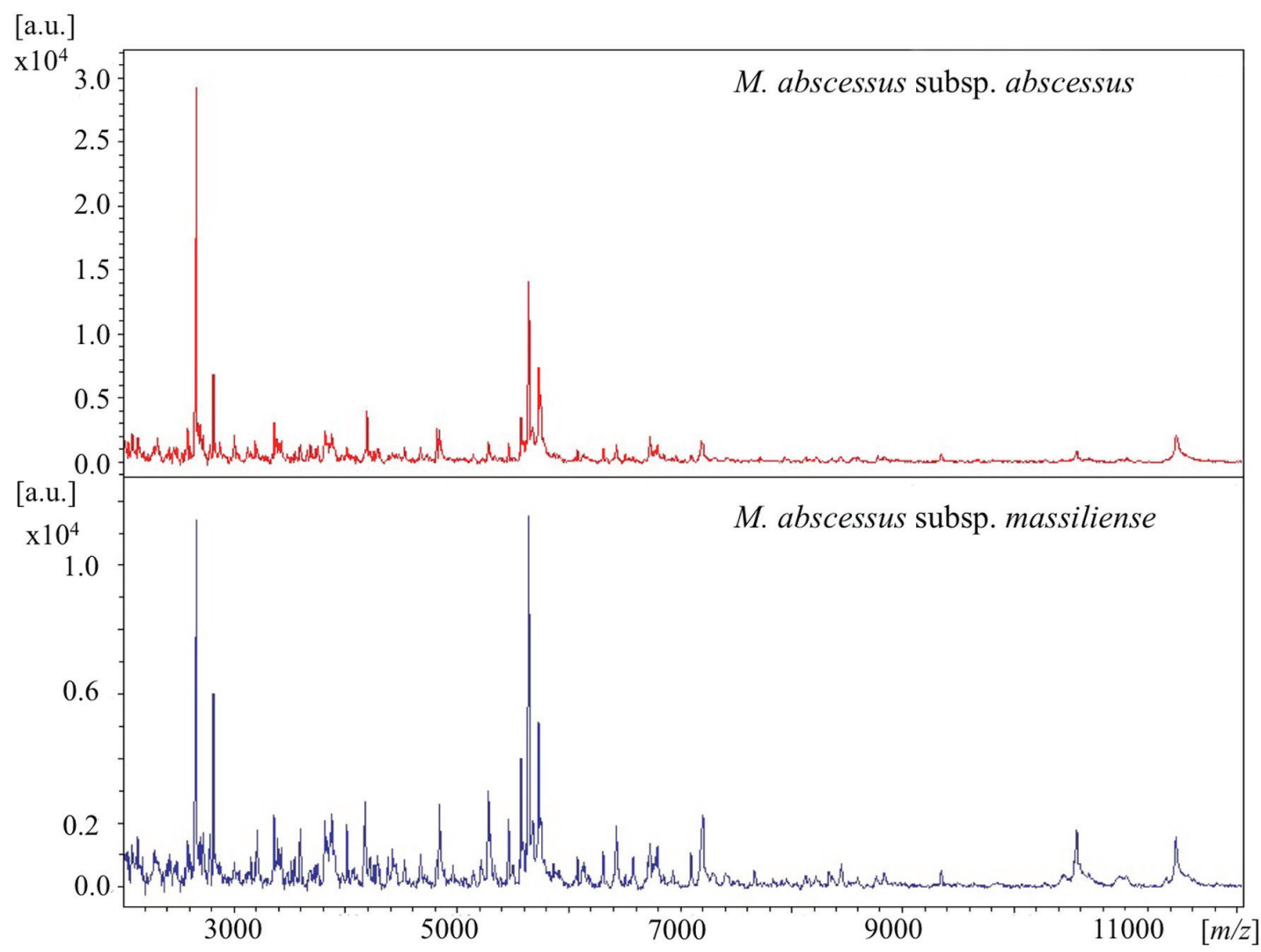

Figure 2. Representative MALDI-TOF spectra of M. abscessus subsp. abscessus and M. abscessus subsp. massiliense (2,000-12,000 m/z). MALDI-TOF, Matrix-assisted laser desorption ionization-time of flight mass spectrometry.

codon (R7stop), have been previously identified in the findings of a case report (40). The data also highlight the importance of the 5 ' end of the erm(41) gene. With rare exceptions, such as the presence of a full-length erm(41) gene $(41,42)$, M. abscessus subsp. massiliense is generally not associated with inducible macrolide resistance because most strains harbor a truncated $\operatorname{erm}(41)$ gene $(4,21,43)$. Interestingly, in the present study, one strain (no. 9626) showed a low initial MIC but the MIC 


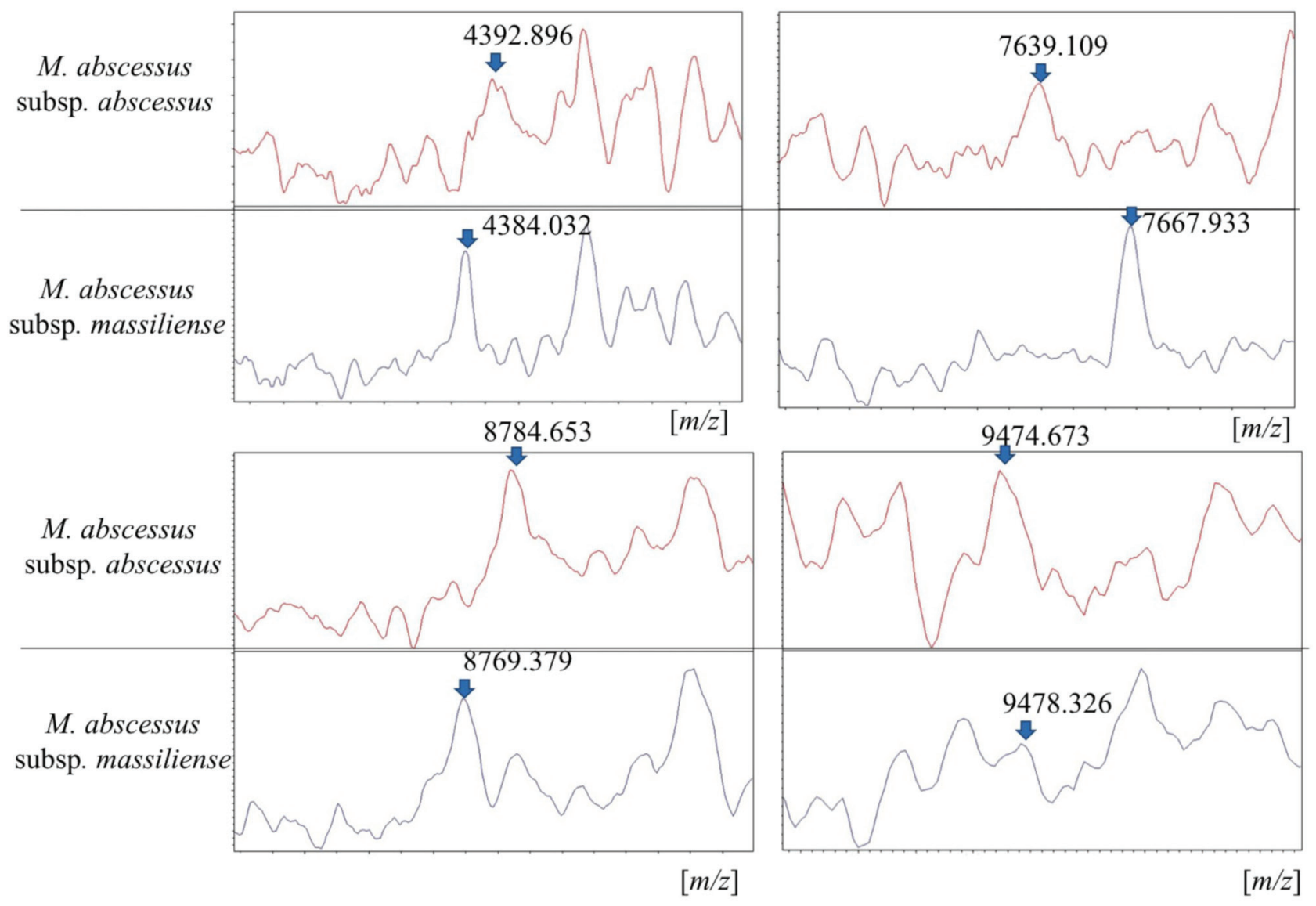

Figure 3. Eight peaks differentiate the two M. abscessus complex subspecies. The diagram shows the relative intensity (\%) against the mass-to-charge ratio values of the discriminating peak regions of MALDI-TOF averaged mass spectral profiles for each subspecies. MALDI-TOF, Matrix-assisted laser desorption ionization-time of flight mass spectrometry.

increased 4-fold by 14 days. This observation suggests that mechanisms not involving erm(41) can cause inducible resistance. No strains that simultaneously harbored both a $r r l$ mutation and nucleotide T28 in erm(41) were detected in this study; however, numerous studies have revealed that both resistance mechanisms can occur concurrently $(36,38)$, and that a functional erm(41) gene does not exclude selection for $r r l$ mutations (44). Transcriptome analysis of M. abscessus complex revealed the presence of several novel open reading frames, which could be activated by stressful conditions, such as hypoxia, to persist (45). Therefore, in addition to antimicrobial resistance genes, pathogenetic gene alterations should also be investigated in the future.

As described above, the two subspecies of the M.abscessus complex were found, which is consistent with the results of other studies showing the predominance of M. abscessus subsp. abscessus and M. abscessus subsp. massiliense in East Asia $(11,16,21,24)$. Data from the current study indicate that the principal difference between the two subspecies was the size of the erm(41) gene. Several studies have revealed the presence of a complete erm(41) gene in M. abscessus subsp. massiliense strains $(41,42)$ and a truncated erm(41) gene in M. abscessus subsp. bolletii (36), suggesting that M. abscessus subsp. massiliense acquired a full-length erm(41) gene by horizontal transfer from M. abscessus subsp. abscessus or M. abscessus subsp. bolletii, and that a truncated erm(41) gene was transferred from M. abscessus subsp. massiliense to
M. abscessus subsp. bolletii. Additionally, a change at position 28T>C has been reported in M. abscessus subsp. bolletii (21). Therefore, although horizontal gene transfer between subspecies is probably quite rare, erm(41) PCR is not proposed as the best way to differentiate M.abscessus complex subspecies. However, erm(41) PCR can be easily and efficiently used for the prediction of sensitivity to clarithromycin in the M. abscessus complex. The phenotypic function of a putative erm(41) gene is important for the clinician from a strictly pragmatic standpoint. Likewise, M. abscessus complex subspecies should be categorized based on the presence of a functional erm(41) gene and macrolide sensitivity especially if horizontal gene transfer increases in the future.

In conclusion, the present study demonstrates the features of M. abscessus subsp. abscessus and M. abscessus subsp. massiliense, isolated in the Tokyo-Yokohama area. No strains of M. abscessus subsp. bolletii were detected. It was not possible to differentiate the two subspecies by clinical features in pulmonary infection, and results of mass spectrometry analysis of both these subspecies were highly similar; however, it is possible to predict clarithromycin susceptibility in strains of the two species by PCR amplification of the erm(41) gene. This is a simple and useful method that can be carried out routinely in hospital laboratories, and is recommended to predict inducible resistance to macrolides before determining the MICs, which requires 14 days of incubation for M. abscessus complex subspecies. 


\section{Acknowledgements}

The authors would like to thank Mr. Sadahiro Ichimura and Mr. Hidetoshi Yamamoto (BML Inc., Saitama, Japan) for their microbiological techniques and Ms. Azumi Fujinaga (Bruker Japan K.K., Yokohama, Japan) for help with the mass spectra analysis.

\section{Funding}

This work was supported by Grant-in-Aid for Scientific Research(C) (grant no. 17K09021).

\section{Availability of data and materials}

The datasets used and/or analyzed during the current study are available from the corresponding author on reasonable request.

\section{Authors' contributions}

AM, FY, TF, YY and YS examined and cared for the patients. AM, FY, TF, YY, YS and KF developed the concept, designed the experiments and analyzed the data. AM wrote the manuscript with contributions from all authors, who commented on it at all stages.

\section{Ethics approval and consent to participate}

Official approval for the study was obtained in advance from the Ethics Committee for Research at Showa University (approval nos. 371 and 2016127). Informed consent was waived due to the retrospective nature of the study.

\section{Patient consent for publication}

Not applicable.

\section{Competing interests}

The authors declare that they have no competing interests.

\section{References}

1. Namkoong H, Kurashima A, Morimoto K, Hoshino Y, Hasegawa N, Ato M and Mitarai S: Epidemiology of pulmonary nontuberculous Mycobacterial disease, Japan. Emerg Infect Dis 22: 1116-1117, 2016.

2. Lee MR, Sheng WH, Hung CC, Yu CJ, Lee LN and Hsueh PR Mycobacterium abscessus complex infections in humans. Emerg Infect Dis 21: 1638-1646, 2015.

3. Wallace RJ, Meier A, Brown BA, Zhang Y, Sander P, Onyi GO and Böttger EC: Genetic basis for clarithromycin resistance among isolates of Mycobacterium chelonae and Mycobacterium abscessus. Antimicrob Agents Chemother 40: 1676-1681, 1996.

4. Nash KA, Brown-Elliott AB and Wallace RJ Jr: A Novel gene, erm(41), confers inducible macrolide resistance to clinical isolates of Mycobacterium abscessus but is absent from mycobacterium chelonae. Antimicrob Agents Chemother 53: 1367-1376, 2009.

5. Teng SH, Chen CM, Lee MR, Lee TF, Chien KY, Teng LJ and Hsueh PR: Matrix-assisted laser desorption ionization-time of flight mass spectrometry can accurately differentiate between Mycobacterium masilliense (M. abscessus subspecies bolletti) and M. abscessus (Sensu Stricto). J Clin Microbiol 51: 3113-3116, 2013.
6. Fangous MS, Mougari F, Gouriou S, Calvez E, Raskine L, Cambau E, Payan C and Hery-Arnaud G: Classification algorithm for subspecies identification within the Mycobacterium abscessus species, based on matrix-assisted laser desorption ionization-time of flight mass spectrometry. J Clin Microbiol 52: 3362-3369, 2014.

7. Panagea T, Pincus DH, Grogono D, Jones M, Bryant J, Parkhill J, Floto RA and Gilligan P: Mycobacterium abscessus complex identification with matrix-assisted laser desorption ionization-time of flight mass spectrometry. J Clin Microbiol 53: 2355-2358, 2015.

8. Suzuki H, Yoshida S, Yoshida A, Okuzumi K, Fukusima A and Hishinuma A: A novel cluster of Mycobacterium abscessus complex revealed by matrix-assisted laser desorption ionization-time-of-flight mass spectrometry (MALDI-TOF MS). Diagn Microbiol Infect Dis 83: 365-370, 2015.

9. Buckwalter SP, Olson SL, Connelly BJ, Lucas BC, Rodning AA, Walchak RC, Deml SM, Wohifiel SL and Wengenack NL: Evaluation of matrix-assisted laser desorption ionization-time of flight mass spectrometry for identification of Mycobacterium species, Nocardia species, and other aerobic actinomycetes. J Clin Microbiol 54: 376-384, 2016.

10. Kehrmann J, Wessel S, Murali R, Hampel A, Bange FC, Buer J and Mosel F: Principal component analysis of MALDI TOF MS mass spectra separates M. abscessus (sensu stricto) from $\mathbf{M}$. massiliense isolates. BMC Microbiol 16: 24, 2016.

11. Harada T, Akiyama Y, Kurashima A, Nagai H, Tsuyuguchi K, Fujii T, Yano S, Shigeto R, Kuraoka T, Kajiki A, et al: Clinical and microbiological differences between Mycobacterium abscessus and Mycobacterium massiliense lung diseases. JClin Microbiol 50: 3556-3561, 2012.

12. Fukuchi K, Hagiwara T, Nakamura K, Ichimura S, Tatsumi K and Gomi K: Identification of the regulatory region required for ubiquitination of the cyclin kinase inhibitor, p21. Biochem Biophys Res Commun 293: 120-125, 2002.

13. Woods GL, Brown-Elliott BA, Conville PS, Desmond EP, Hall GS and Lin G: Susceptibility testing of Mycobacteria, Nocardia and other aerobic actinomycetes; Approved Standard-Second edition. Clin Lab Stand Inst 26: 1-61, 2011.

14. Macheras E, Roux AL, Bastian S, Leao SC, Palaci M, Tardy VS, Gutierrez C, Richter E, Gerdes SR, Pfyffer G, et al: Multilocus sequence analysis and rpoB sequencing of Mycobacterium abscessus (sensu lato) strains. J Clin Microbiol 49: 491-499, 2011.

15. Griffith DE, Aksamit T, Brown-Elliott BA, Catanzaro A, Daley C, Gordin F, Holland SM, Horsburgh R, Huitt G, Iademarco MF, et al: An official ATS/IDSA statement: Diagnosis, treatment, and prevention of nontuberculous mycobacterial diseases. Am J Respir Crit Care Med 175: 367-416, 2007.

16. Yoshida S, Tsuyuguchi K, Suzuki K, Tomita M, Okada M, Hayashi S, Iwamoto $\mathrm{T}$ and Saito $\mathrm{H}$ : Further isolation of Mycobacterium abscessus subsp. abscessus and subsp. bolletii in different regions of Japan and susceptibility of these isolates to antimicrobial agents. Int J Antimicrob Agents 42: 226-231, 2013.

17. Nakanaga K, Sekizuka T, Fukano H, Sakakibara Y, Takeuchi F, Wada S, Ishii N, Makino M, Kuroda M and Hoshino Y: Discrimination of Mycobacterium abscessus subsp. massiliense from Mycobacterium abscessus subsp. abscessus in clinical isolates by multiplex PCR. J Clin Microbiol 52: 251-259, 2014.

18. Yoshida S, Arikawa K, Tsuyuguchi K, Kurashima A, Harada T, Nagai H, Suzuki K, Iwamoto T and Hayashi S: Investigation of the population structure of Mycobacterium abscessus complex strains using 17-locus variable number tandem repeat typing and the further distinction of mycobacterium massiliense hsp 65 genotypes. J Med Microbiol 64: 254-261, 2015.

19. Kusuki M, Osawa K, Arikawa K, Tamura M, Shigemura K, Shirakawa T, Nakamura T, Nakamachi Y, Fujisawa M, Saegusa J and Tokimatsu I: Determination of the antimicrobial susceptibility and molecular profile of clarithromycin resistance in the Mycobacterium abscessus complex in Japan by variable number tandem repeat analysis. Diagn Microbiol Infect Dis 91: 256-259, 2018.

20. Koh WJ, Jeon K, Lee NY, Kim BJ, Kook YH, Lee SH, Park YK, Kim CK, Shin SJ, Huitt GA, et al: Clinical significance of differentiation of Mycobacterium massiliense from Mycobacterium abscessus. Am J Respir Crit Care Med 183: 405-410, 2011.

21. Kim HY, Kim BJ, Kook Y, Yun YJ, Shin JH, Kim BJ and Kook YH: Mycobacterium massiliense is differentiated from Mycobacterium abscessus and mycobacterium bolletii by erythromycin ribosome methyltransferase gene (erm) and clarithromycin susceptibility patterns. Microbiol Immunol 54: 347-353, 2010. 
22. Kim BJ, Yi SY, Shim TS, Do SY, Yu SK, Park YG, Kook YH and Kim BJ: Discovery of a novel hsp65 genotype within Mycobacterium massiliense associated with the rough colony morphology. PLoS One 7: e38420, 2012.

23. Kim SY, Kang YA, Bae IK, Yim JJ, Park MS, Kim YS, Kim SK, Chang J and Jeong SH: Standardization of multilocus sequence typing scheme for Mycobacterium abscessus and Mycobacterium massiliense. Diagn Microbiol Infect Dis 77: 143-149, 2013.

24. Luo L, Li B, Chu H, Huang D, Zhang Z, Zhang J, Gui T, $\mathrm{Xu}$ L, Zhao L, Sun X and Xiao H: Characterization of Mycobacterium abscessus subtypes in Shanghai of China: Drug sensitivity and bacterial epidemicity as well as clinical manifestations. Medicine (Baltimore) 95: e2338, 2016.

25. Koh WJ, Jeong BH, Kim SY, Jeon K, Park KU, Jhun BW, Lee H, Park HY, Kim DH, Huh HJ, et al: Mycobacterial characteristics and treatment outcomes in Mycobacterium abscessus lung disease. Clin Infect Dis 64: 309-316, 2017.

26. Davidson RM, Hasan NA, Reynolds PR, Totten S, Garcia B, Levin A, Ramamoorthy P, Heifets L, Daley CL and Strong M: Genome sequencing of Mycobacterium abscessus isolates from patients in the United States and comparisons to globally diverse clinical strains. J Clin Microbiol 52: 3573-3582, 2014.

27. Leao SC, TortoliE, Viana-Niero C, Ueki SY,Lima KV, Lopes ML Yubero J, Menendez MC and Garcia MJ: Characterization of mycobacteria from a major Brazilian outbreak suggests that revision of the taxonomic status of members of the Mycobacterium chelonae-M. abscessus group is needed. J Clin Microbiol 47: 2691-2698, 2009.

28. Tan JL, Khang TF, Ngeow YF and Choo SW: A phylogenomic approach to bacterial subspecies classification: Proof of concept in Mycobacterium abscessus. BMC Genomics 14: 879, 2013.

29. Kikuchi T, Watanabe A, Gomi K, Sakakibara T, Nishimori K Daito H, Fujimura S, Tazawa R, Inoue A, Ebina M, et al: Association between mycobacterial genotypes and disease progression in Mycobacterium avium pulmonary infection. Thorax 64: 901-907, 2009.

30. Wong YL, Ong CS and Ngeow YF: Molecular typing of Mycobacterium abscessus based on tandem-repeat polymorphism. J Clin Microbiol 50: 3084-3088, 2012.

31. Mougari F, Raskine L, Ferroni A, Marcon E, Sermet-Gaudelus I, Veziris N, Heym B, Gaillard JL, Nassif X and Cambau E: Clonal relationship and differentiation among Mycobacterium abscessus isolates as determined using the semiautomated repetitive extragenic palindromic sequence PCR-based diversilab system. J Clin Microbiol 52: 1969-1977, 2014.

32. Kim HY, Kook Y, Yun YJ, Park CG, Lee NY, Shim TS, Kim BJ and Kook YH: Proportions of Mycobacterium massiliense and Mycobacterium bolletii strains among Korean Mycobacterium chelonae-Mycobacterium abscessus group isolates. J Clin Microbiol 46: 3384-3390, 2008.

33. Kim BJ, Kim GN, Kim BR, Shim TS, Kook YH and Kim BJ: Phylogenetic analysis of Mycobacterium massiliense strains having recombinant $\mathrm{rpoB}$ gene laterally transferred from Mycobacterium abscessus. PLoS One 12: e0179237, 2017.

34. Steindor M, Nkwouano V, Stefanski A, Stuehler K, Loerger TR, Bogumil D, Jacobsen M, Mackenzie CR and Kalscheuer R: A proteomics approach for the identification of species-specific immunogenic proteins in the Mycobacterium abscessus complex. Microbes Infect: Nov 13, 2018 (Epub ahead of print). doi: 10.1016/j.micinf.2018.10.006.
35. Lee SH, Yoo HK, Kim SH, Koh WJ, Kim CK, Park YK and Kim HJ: Detection and assessment of clarithromycin inducible resistant strains among Korean Mycobacterium abscessus clinical strains: PCR methods. J Clin Lab Anal 28: 409-414, 2014.

36. Rubio M, March F, Garrigó M, Moreno C, Español M and Coll P: Inducible and acquired clarithromycin resistance in the Mycobacterium abscessus complex. PLoS One 10: e0140166, 2015.

37. Bastian S, Veziris N, Roux AL, Brossier F, Gaillard JL, Jarlier V and Cambau E: Assessment of clarithromycin susceptibility in strains belonging to the Mycobacterium abscessus group by erm(41) and rrl sequencing. Antimicrob Agents Chemother 55: 775-781, 2011.

38. Mougari F, Bouziane F, Crockett F, Nessar R, Chau F, Veziris N, Sapriel G, Raskine L and Cambau E: Selection of resistance to clarithromycin in Mycobacterium abscessus subspecies. Antimicrob Agents Chemother 61: pii: e00943-16, 2016.

39. Kudoh S and Keicho N: Diffuse panbronchiolitis. Clin Chest Med 33: 297-305, 2012.

40. Kim SY, Shin SJ,Jeong BHandKoh WJ: Successful antibiotic treatment of pulmonary disease caused by Mycobacterium abscessus subsp. abscessus with C-to-T mutation at position 19 in erm(41) gene: Case report. BMC Infect Dis 16: 207, 2016.

41. Shallom SJ, Gardina PJ, Myers TG, Sebastian Y, Conville P, Calhoun LB, Tettelin H, Olivier KN, Uzal G, Sampaio EP, et al: New rapid scheme for distinguishing the subspecies of the Mycobacterium abscessus group and identifying Mycobacterium massiliense isolates with inducible clarithromycin resistance. J Clin Microbiol 51: 2943-2949, 2013

42. Brown-Elliott BA, Vasireddy S, Vasireddy R, Iakhiaeva E, Howard ST, Nash K, Parodi N, Strong A, Gee M, Smith T and Wallace RJ Jr: Utility of sequencing the erm(41) gene in isolates of Mycobacterium abscessus subsp. abscessus with low and intermediate clarithromycin MICs. J Clin Microbiol 53: 1211-1215, 2015.

43. Yoshida S, Tsuyuguchi K, Suzuki K, Tomita M, Okada M, Shimada R and Hayashi S: Rapid identification of strains belonging to the Mycobacterium abscessus group through erm(41) gene pyrosequencing. Diagn Microbiol Infect Dis 79: 331-336, 2014.

44. Maurer FP, Rüegger V, Ritter C, Bloemberg GV and Böttger EC: Acquisition of clarithromycin resistance mutations in the $23 \mathrm{~S}$ rRNA gene of Mycobacterium abscessus in the presence of inducible erm(41). J Antimicrob Chemother 67: 2606-2611, 2012.

45. Miranda-CasoLuengo AA, Staunton PM, Dinan AM, Lohan AJ and Loftus BJ: Functional characterization of the Mycobacterium abscessus genome coupled with condition specific transcriptomics reveals conserved molecular strategies for host adaptation and persistence. BMC Genomics 17: 553, 2016.

This work is licensed under a Creative Commons Attribution-NonCommercial-NoDerivatives 4.0 International (CC BY-NC-ND 4.0) License. 